\title{
Performance Characteristics of an Adaptive Controller Based on Least-Mean-Square Filters
}

Rajiv S. Mehta and Shmuel J. Merhav

(NASA-TH-88359) FERFCAMANCE CHAKACTERISTICS

CF AN ALAETIVE CCATRCIIER EASED CN

IEAST-MEAN-SQUARE FIIIEBS (NASA) $14 \mathrm{p}$

$$
\text { CSCL 128 Unclas }
$$

G3/66 43512

September 1986 
NASA Technical Memorandum 88359

\section{Performance Characteristics of an Adaptive Controller Based on Least-Mean-Square Filters}

Rajiv S. Mehta,

Shmuel J. Merhav, Ames Research Center, Moffett Field, California

September 1986

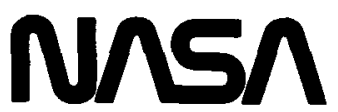

National Aeronautics and

Space Administration

Ames Research Center

Moffett Field, California 94035 
Rajiv S. Mehta ${ }^{\dagger}$ and S. J. Merhav

NASA Ames Research Center, Moffett Field, California

\section{Abstract}

A closed-loop, adaptive-control scheme that uses a least-mean-square filter as the controller model is presented, along with simulation results that demonstrate the excellent robustness of this scheme. It is shown that the scheme adapts very well to unknown plants, even those that are marginally stable, responds appropriately to changes in plant parameters, and is not unduly affected by additive noise. A heuristic argument for the conditions necessary for convergence is presented. Potential applications and extensions of the scheme are also discussed.

\section{Introduction}

An adaptive controller is a controller that can change its behavior to maintain good control in response to changes in the process and inputs. One common approach to adaptive control is called Model Reference Adaptive Control (MRAC), in which the specifications are given in terms of a reference model which determines how the plant output ideally should respond to the command signal. In most MRAC schemes the controller is modeled as a differential equation in one of the common forms (i.e., transfer function or state space) with parameters that are adjusted to modify its behavior. A substantial advantage of such parametric modeling is that relatively fast convergence of the adaptive algorithm can be achieved as a result of the small number of parameters involved. However, this approach requires that enough is known about the plant to construct a valid model. The resulting controller design can be quite complex and its performance strongly dependent on the assumed form of the plant model. 1 An excellent survey of adaptive control which discusses many of the problems of the parametric approach is found in Ref. 2. Concerns about the robustness of these schemes has also recently been expressed in Refs. 3 and 4.

A different approach involves adaptive control schemes based on impulse response modeling. Richalet 5 modeled multivariable industrial processes with impulse responses and used these models off-line to determine the results of various possible input sequences. Widrow used what

*This paper, AIAA-86-2160, was presented at the AIAA Guidance, Navigation and Control Conference in Williamsburg, Virginia, on August 18-20, 1986.

$t_{\text {Research Scientist. Member AIAA. }}$

*Professor, Department of Aeronautical Engineering, Israel Institute of Technology, Haifa, Israel. Member AIAA. is known as a least-mean-square (LMS) filter in an essentially open-loop MRAC scheme. The LMS filter, which determines the coefficients of a moving-average model using a gradient algorithm, is a modeling approach that has been widely used in the communication systems field. ${ }^{7}$ With this approach one attempts to produce an approximate impulse response of the desired system and not its exact mathematical form in terms of the coefficients of a differential equation. This approach can be used even when little is known about the physical process. The use of the LMS filter in an adaptive scheme provides robustness and insensitivity to unmodeled modes. The penalty is slower convergence because of the comparatively large number of parameters.

Previous MRAC schemes which used LMS filters have been essentially in open-loop or off-line configurations. Closed-loop control is desired because of its ability to suppress both disturbances and the effects of abrupt changes in plant parameters. One of the principal factors that has hampered the development of closed-loop adaptive control is the interaction between the feedback of the learning process and that of the control signals. This interaction greatly complicates the analysis underlying the design of dependable control systems. A closed-10op MRAC scheme based on LMS filters have recently been proposed ${ }^{8}$ that has been shown to work well with a wide range of plants, even for processes that are barely stable.

This paper presents a further study that extends the work presented in Ref. 8. The adaptive algorithm is first described, followed by a demonstration of its performance. Next, the heuristic arguments are provided to analyze its convergence properties and simulation results demonstrate its robustness. Further simulations show the scheme's tolerance for and response to changes in plant characteristics and to noise. Finally, the potential applications of this adaptive controller are discussed.

\section{The Concept}

The LMS filter, shown in Fig. 1, consists of an input signal $x(t)$ that is sampled and processed by a set of delays (a "tapped delay line," where $g$ is a delay operator), variable weights $w_{i}$ which multiply the signals at the delay line taps, a summer $\Sigma$ where the weighted signals are combined to form the filter output $y$ and an adaptation algorithm to adjust the weights to make the filter output match a desired signal. 


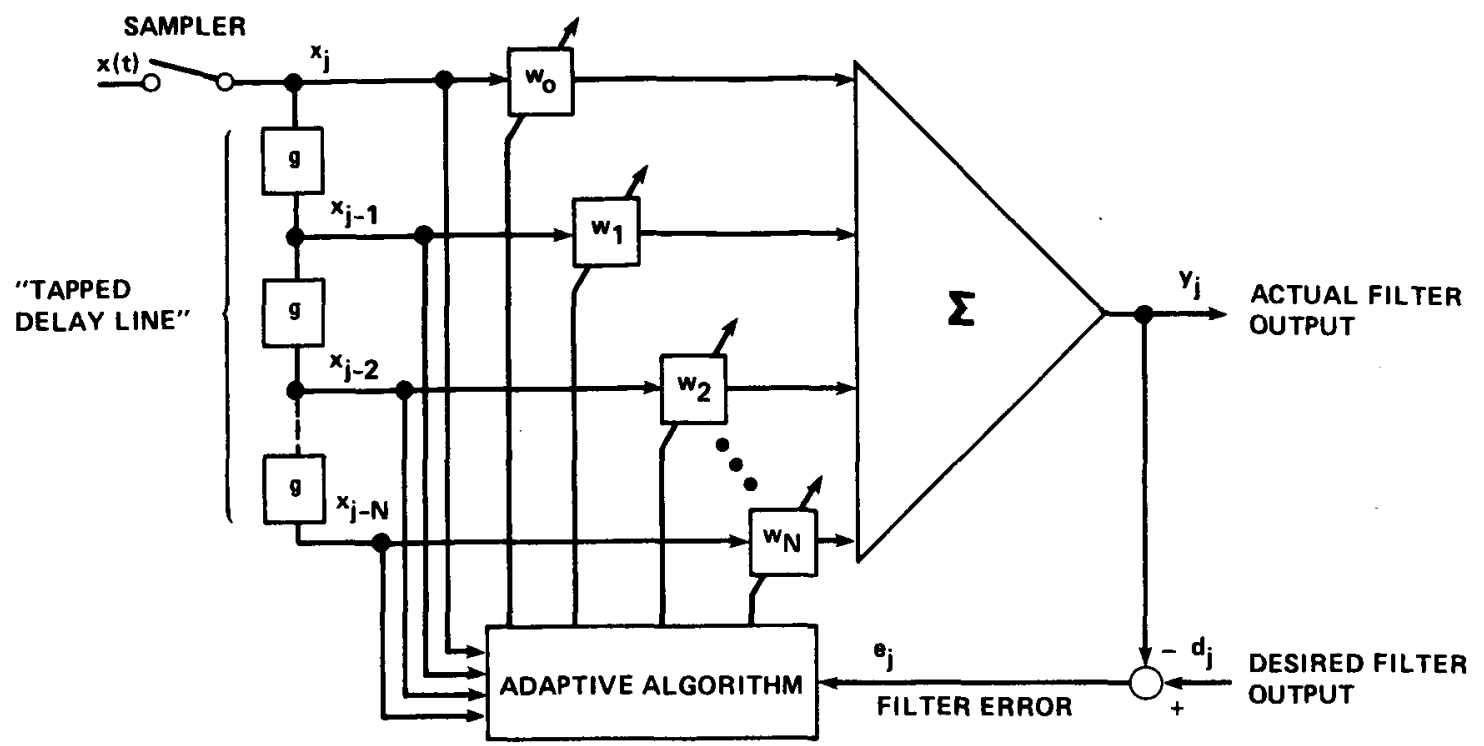

Fig. 1 The LMS filter.

The filter error equation, in discrete form, where $d_{j}$ is the desired output, $y_{j}$ is the actual filter output and $w$ is the weight vector, is

$$
e_{j}=d_{j}-y_{j}=d_{j}-x_{j}^{T} w_{j}
$$

where

$$
x_{j}=\left[\begin{array}{c}
x_{j} \\
x_{j-1} \\
\vdots \\
x_{j-N}
\end{array}\right]
$$

The weights are adjusted using a gradient algorithm to minimize the square of the filter error

$$
\begin{aligned}
& \underline{w}_{j+1}=\underline{w}_{j}+\Delta \underline{w}_{j} \\
& \underline{\Delta w}_{-J}=-\left.\mu \frac{\partial e_{j}^{2}}{\partial \underline{w}}\right|_{\underline{w}=\underline{W}}=-\left.2 \mu e_{j} \frac{\partial e_{j}}{\partial \underline{w}}\right|_{\underline{w}=\underline{J}}
\end{aligned}
$$

In the following, "gradient" will refer to $\left(\partial e_{j}^{2} / \partial w\right)$, while "error gradient" will refer to $\left(a e_{j} / \partial \bar{w}\right)$. The term $\mu$ is a gain factor that is chosen to assure the stability of the gradient algorithm. Using Eq. (1) the error gradient is

$$
\left.\frac{\partial e j}{\partial \underline{w}}\right|_{w=-j}=-\underline{j}
$$

and

$$
\Delta w_{j}=2 \mu e_{j} x_{j}
$$

The properties of this filter have been studied extensively 7,9 and are briefly summarized here. Eq. (3) represents a difference equation which can be analyzed for stability and convergence. A sufficient condition for stable convergence is $\left(0<\mu<1 / x_{1} x_{1}\right)$. In the implementation of the LMS filter we let

$$
\mu=\frac{1}{F \underline{x}_{j}^{T}-j} \quad(F>1)
$$

and use $F$ as a design parameter. The theoretical settling time of the filter can be shown to be $(\mathrm{N}+1) \mathrm{F}$ iterations, and the accuracy of the convergence is related to $1 / F$, so the parameter $F$ may be used to balance the conflicting requirements of speed and accuracy. Note that by choosing $u$ in this manner, the parameter change, Eq. (6), is normalized with respect to the input power.

A necessary condition for the convergence and stability of the LMS filter is for the error equation, Eq. (1), to be linear in the weight vector. This requirement poses a challenge to the design of a closed-10op adaptive controller using LMS filters because the desired filter output $d$ can become a function of the filter weights, possibly in a nonlinear manner. Other considerations in the development of a satisfactory controller are the realizability of the error gradient and whether the controller is determined "directly" or "indirectly." Indirectly means that the controller is determined from an identified plant model, and therefore involves at least one additional stage beyond that required for directly 


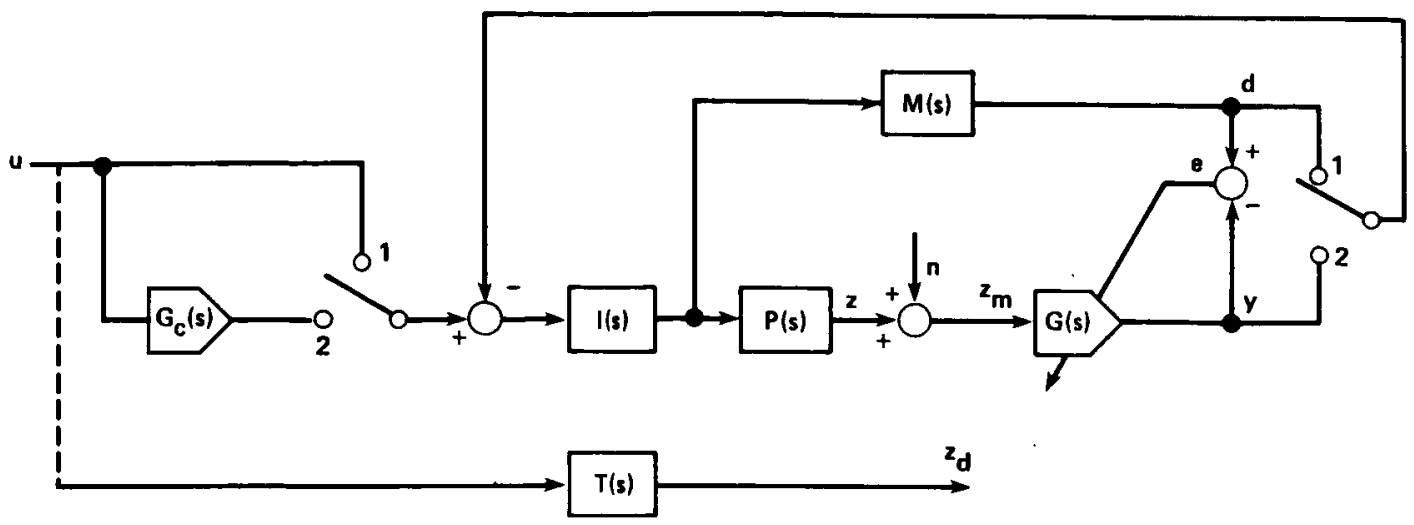

Fig. 2 The system configuration.

determining the controller. As was shown in Ref. 8, a closed-loop configuration that is stable, realizable, and direct is extremely difficult to design. The configuration presented in this paper yields an expression for the closedloop gradient which strongly indicates global and uniform convergence properties.

Figure 2 shows the system configuration: $P(s)$ is the unknown plant; $M(s)$ is the open-loop reference model; $I(s)$ is an integrator; $G(s)$ is the adaptive controller structured as an LMS filter; $G_{C}(s)$ is an exact, current copy of the filter $G(s)$; and $T(s)$ is the desired closed-loop reference model, obtained directly from $M(s)$, and is used for comparison only. Inputs to the system are $u$ the desired input, and $n$ which is additive noise. It is easily verified that, with respect to the filter the process and measurement noise are equivalent, ${ }^{8}$ and therefore one noise source will suffice for the simulations. Notice that the configuration has two different modes of operation. When the switches are in Position 1 , $G(s)$ and $G_{c}(s)$ are not in loop, and therefore do not affect the input or output of the plant. This state is called the "open-loop" state. With the switches in Position 2, $G(s)$ and $G_{c}(s)$ form the controller for the plant. This state will be referred to as the "closed-loop" state.

The adaptation process has to adjust $G(s)$ so that $P(s) G(s) \rightarrow M(s)$. Essentially, the only a priori information needed for the unknown plant is that it be stable and minimum phase. The LMS filter is represented in continuous time as

$$
G(s)=\sum_{i=0}^{N} g^{i}(s) w_{i}
$$

where $g^{1}(s)$ represents a delay of $i$ time steps. The choice of the number of weights $N$ and the value of the time delays are determined a priori from the characteristics of the reference model $M(s)$. Rules for choosing these quantities are given in Ref. 8.
In the open loop state, the filter error is given by*

$$
e(t)=\frac{[M(s)-G(s) P(s)] I(s)}{1+M(s) I(s)} u(t)
$$

which is linear in $G(s)$, and therefore in $w$. The ith error gradient component is

$$
\begin{aligned}
\frac{\partial e(t)}{\partial w_{i}} & =\frac{\partial e(t)}{\partial G(s)} \frac{\partial G(s)}{\partial w_{i}}=\frac{-P(s) I(s)}{1+M(s) I(s)} g^{i}(s) u(t) \\
& =-g^{i}(s) z(t) \quad(i=0, \ldots ., N)
\end{aligned}
$$

The signal $g^{i}(s) z(t)$ is the signal $z(t)$ delayed $i$ time steps and is realizable. So, both the linearity and realizability conditions are satisfied for the open-loop configuration.

In the closed-loop state, the filter error is given by**

$$
e=\frac{(M-G P) G I}{1+G P I} u
$$

which is not linear in the weights. The error gradient can be shown to be

$$
\frac{\partial e}{\partial w_{i}}=-g^{i} z+\frac{1}{G(1+G P I)} g^{i} e
$$

If the system has converged and $P G=M$, then Eqs. (11) and (12) become

* Presently, it is assumed that the noise is zero. The effect of noise will be discussed later in the paper.

** In the rest of the paper, for brevity, the complex frequency variable $s$ and the dependence on time $t$ will not always be explicitly written (i.e., $P$ will stand for $P(s)$ and $z$ for $z(t))$. 


$$
\begin{gathered}
e=0 \\
\frac{\partial e}{\partial w_{i}}=-g^{i} z
\end{gathered}
$$

Thus, the error gradient assumes its correct openloop form as determined by Eq. (10).

It is evident that the system will not start up properly in the closed-loop state because the error equation, Eq. (11), is not linear in the weights as required. The initial approach to resolve this problem is to start the system in the open-10op state and to switch to the closed-loop state after the filter has converged. After that, if the changes in the plant are moderate the system can reconverge because the gradient, Eq. (14), is approximately correct. The issue of whether the system can be started in the closedloop state will be addressed again shortly.

\section{Performance Demonstration}

This section demonstrates the performance of this adaptive controller. Let the unknown plant be

$$
P(s)=\frac{25}{s^{2}+0.1 s+25}
$$

$P$ has a pair of complex poles with natural frequency $\omega$ of 5 and damping factor $\zeta$ of 0.01 . Let the open-loop reference model be

$$
M(s)=\frac{10}{s^{2}+6.5 s+9}
$$

The relation between the open and closed-1oop reference models is given by

$$
T(s)=\frac{M(s)}{s+M(s)}=\frac{10}{s^{3}+6.5 s^{2}+9 s+10}
$$

The filter $G$ has 61 weights $(N=60)$ with a tap spacing of $0.04 \mathrm{sec}$, and $F$ is chosen to be 10 . Ideally, when the system has adapted perfectly, GP should be equal to $M$, and therefore

$$
G(s)=\frac{M(s)}{P(s)}=\frac{0.4\left(s^{2}+0.1 s+25\right)}{\left(s^{2}+6.5 s+9\right)}
$$

Example 1: The actual results of using the adaptive controller with this plant and reference model are shown in Figs. 3 through 5 . These figures were generated from a run of 1200 iterations (48 sec) with the first 600 iterations being in the open-loop state and the rest in the closedloop state. The final filter weights representing the finite impulse response (FIR) are shown in Fig. 3 along with the infinite impulse response

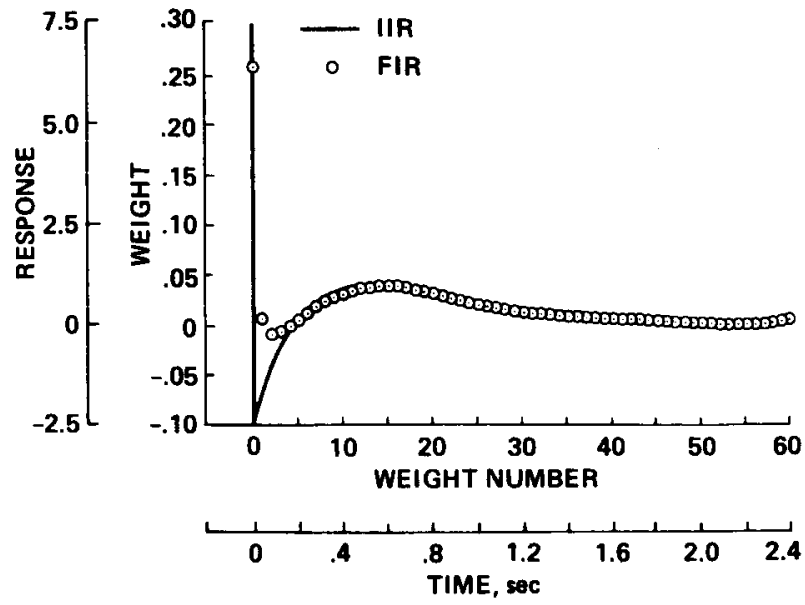

Fig. 3 Estimated FIR and IIR of $G(s)$, Example 1.

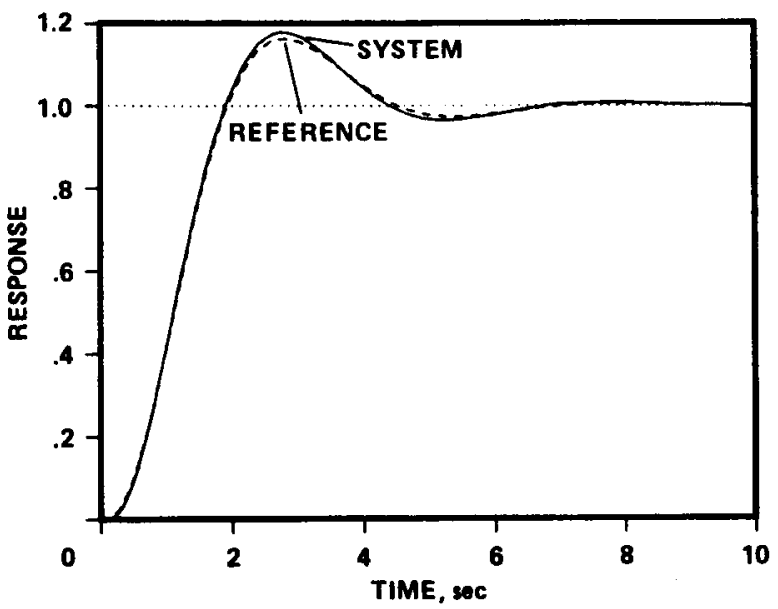

Fig. 4. Step response of adapted system and reference model $T(s)$, Example 1 .

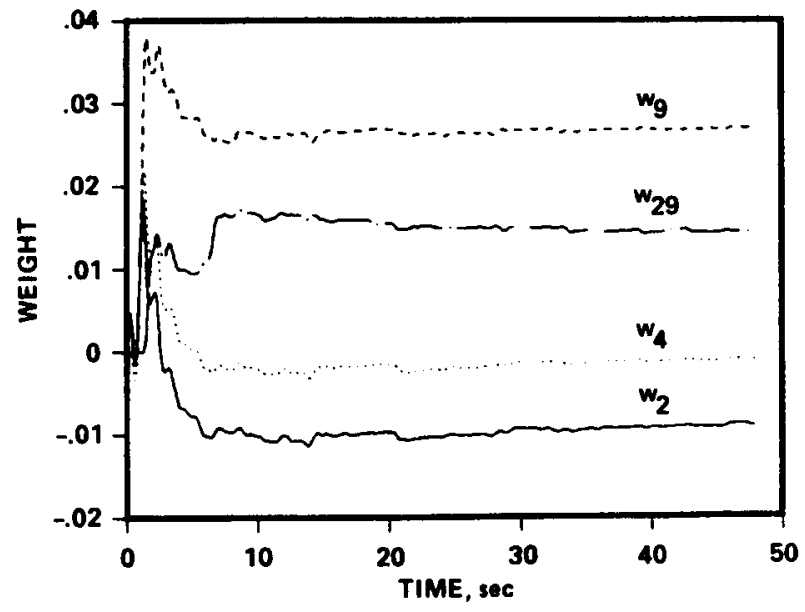

Fig. 5 Time history of filter weights, Example 1. 
(IIR) of the ideal $G(s)$ as represented by Eq. (18). The shapes of the FIR and IIR are very similar and one can conclude that the system has adapted well. Figure 4, which verifies this conclusion, shows the step response of the reference model $T(s)$ and that of the adapted system (that is, the step response of the system with the weights frozen at the values obtained at the end of the run). Figure 5 shows the time history of four of the filter weights throughout the duration of the run. The weights had settled after about $10 \mathrm{sec}$ (250 iterations) and after $25 \mathrm{sec}(625$ iterations) they were very steady. This overall settling time agrees well with our expectations $((\mathrm{N}+1) \mathrm{F}=610$ iterations $)$. If Fig. 5 is examined closely it appears that the weights are drifting at the end of the simulation, from 25 to $50 \mathrm{sec}$, but in actuality the controller is simply adjusting to a very small change in the process-there are slight differences in the simulation between the open- and closed-loop states. After this adjustment, that is after the 50 sec mark, the weights are constant. The steadiness of the weights will be apparent in Fig. 9 which accompanies Example 2 further on in the paper.

The system works just as well as in Example 1 when the damping of the second-order plant is varied from $10^{-3}$ to 1 and its relative degree varicd from one to two. The input signal, 1 , used in Example 1, and for all the other examples of the paper, was white noise. Other input sequences such as colored noise and a series of random steps have also been used in simulations with good results.

\section{Convergence Properties}

To understand whether the system can start up and converge completely in its closed-loop state, we again look at the expressions for the error gradient. As was indicated before, the implemented and theoretical gradients become equal once the system has converged. However, the question is how similar the two gradient expressions are before the filter has converged to the correct values. Equation (12) representing the theoretical error gradient can be rewritten in terms of the input $u$ as

$$
\frac{\partial e}{\partial w_{i}}=\frac{M I-G P I(2+G P I)}{(1+G P I)^{2}} g^{i} u \triangleq-D_{T} g^{i} u
$$

The implemented gradient (Eq. (14)) is

$$
\frac{\partial e}{\partial w_{i}}=-g^{i} z
$$

or

$$
\frac{\partial e}{\partial w_{i}}=\frac{-G P I}{(1+G P I)} g^{i} u \triangleq-D_{I} g^{i} u
$$

With these expressions, the following heuristic argument is offered. Equation (4), which expresses the parameter updates, using Eqs. (19) and (21), can be written as

$$
\left[\begin{array}{c}
\Delta w_{0} \\
\Delta w_{1} \\
\vdots \\
\Delta w_{N}
\end{array}\right]_{j}=2 \mu e_{j}\left(D_{T} \text { or } \quad D_{I}\right)\left[\begin{array}{c}
u_{j} \\
u_{j-1} \\
\vdots \\
u_{j-N}
\end{array}\right]
$$

The difference between the implemented and theoretical gradients is the transfer function that filters the signal $g i_{u}$. For the system to converge, the implemented error gradient must "point" in essentially the same direction as the theoretical error gradient. This fact implies that the corresponding elements of the two error gradients must have the same sign, which means that the transfer functions $D_{T}$ and $D_{I}$ must have similar phase responses.

We will look at an example to illustrate this statement. Let the plant and the model be those of Eqs. (15) and (16). The I, as in Eq. (19), stands for the integrator and is

$$
I(s)=1 / s
$$

As a starting condition for the filter, let the weights be set to

$$
G:\left\{\begin{array}{l}
w_{0}=1 \\
w_{i}=0 \quad(i=1, N)
\end{array}\right.
$$

Then the initial value of $G(s)$ is

$$
G(s)=1
$$

Note that the system must be started in closedloop position with at least one nonzero filter weight because otherwise the input signal will not get past the first filter and cannot start up (Fig. 2).

The transfer functions $D_{T}$ and $D_{I}$ can be evaluated using the expressions for $M(s), P(s)$, $I(s)$, and $G(s)$ in accordance with Eqs. (15), (16), (23), and (25). The Bode phase and magnitude plots are shown in Figs. 6 and 7 . The phase plots are very similar, differing at most by $30^{\circ}$ in a small-frequency range, from 0.8 to $3.5 \mathrm{rad} / \mathrm{sec}$. This fact means that the elements of the implemented and theoretical gradients will have the same signs. Thus, the implemented gradient changes the weights in the correct direction so that they will move closer to their correct values, and the two gradients will match even more closely in future iterations. The magnitude plot shows that the implemented gradient is slightly smaller, meaning that the system will converge 


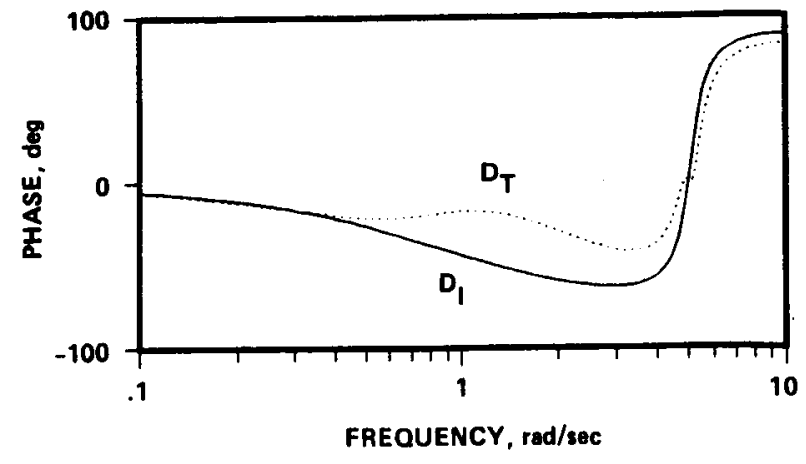

Fig. 6 Phase response of theoretical error gradient, $D_{T}$, and implemented error gradient, $D_{I}$.

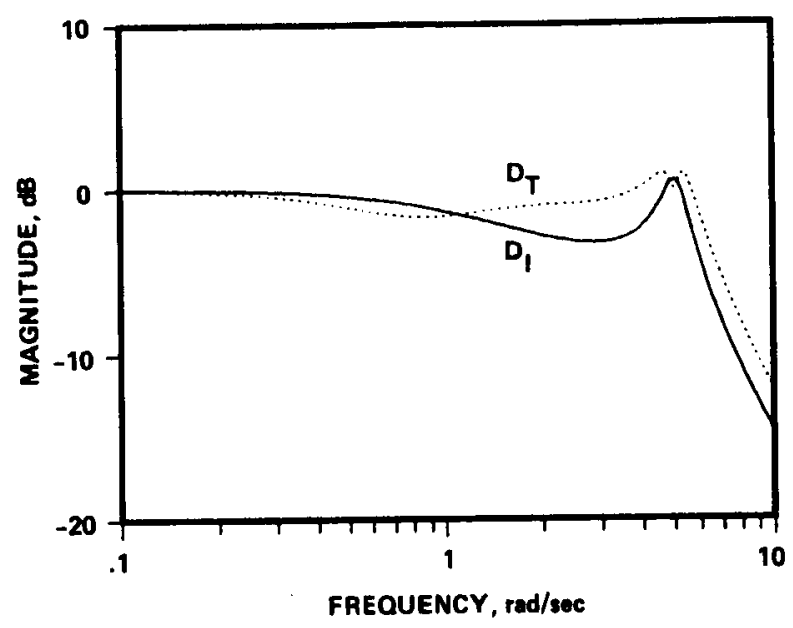

Fig. 7 Magnitude response of theoretical error gradient, $\mathrm{D}_{\mathrm{T}}$, and implemented error gradient, $\mathrm{D}_{\mathrm{I}}$.

more slowly than predicted. This premise is supported by the inspection of Eq. (12).

Example 2: According to this heuristic argument, at least for the example of Eqs. (15) and (16), the system should be able to converge even when started up in the closed-loop configuration given the initial weights of Eq. (24). The simulation results confirm this assertion. Figure 8 shows the resulting step response and Fig. 9 shows the convergence of some of the filter weights. These figures were obtained with the same number of iterations (1200) as those of the previous example. The step response of Fig. 8 is not quite as good as that shown in Fig. 4, and that is because of the slower convergence of the system in the closed-loop state. Nonetheless, the step response of the system in the present example does get as good as that of the previous example given some more time.

A practical way is to start the system in the open-loop state with all the weights set to zero, and then run it for, say, five iterations and switch to the closed-loop mode. Numerous

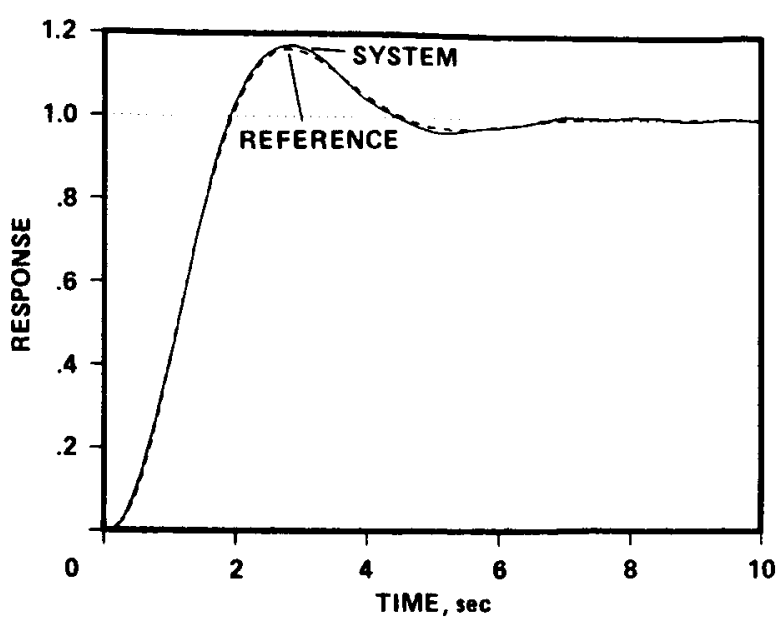

Fig. 8 Step response of adapted system and reference model $\mathrm{T}(\mathrm{s})$, Example 2 .

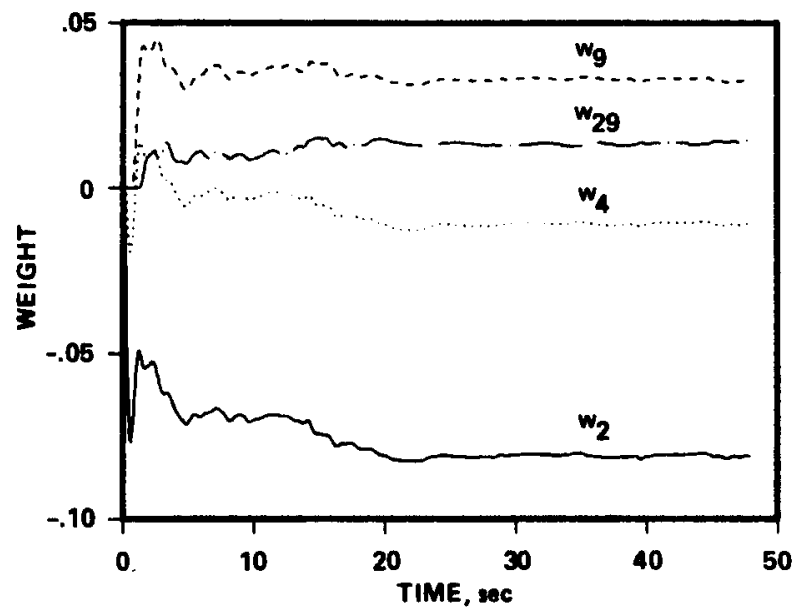

Fig. 9 Time history of filter weights, Example 2.

simulations have confirmed convergence by this method. Although the discussion presented here showed convergence for a speciflc example only, the idea can be extended to a more general test. Given an initial $G$ and the reference model $M$, one can find the range of possible plants $P$ for which the resulting phase plots are similar enough for the algorithm to converge. Conversely, given a range of $P$, one can calculate the range of possible reference models $M$ that assure convergence.

\section{Changes in Plant}

The foregoing demonstrates that the controller adapts well to an unknown plant. It is equally important that an adaptive controller respond appropriately to plant changes that occur after the system has reached its steady state. Because the system will be in the closed-loop state, the previous arguments regarding the inaccuracies in the gradient calculation are still 
pertinent; that is, the allowable variations in plant parameters depend on the effect they have on the phase of $D_{I}$ and $D_{T}$. The performance of this scheme under such situations is demonstrated by the following two examples. For both examples the run of Example 1 was repeated for the first $40 \mathrm{sec}$, at which point a step change in plant parameters was introduced.

Example 3: For this example, the damping ratio of $P(s)$ was changed from $\zeta=0.01$ to $\zeta=0.05$, a five-fold increase. Figure 10 shows the time history of some of the filter weights during this run. We see that after the weights had settled the first time, they adjusted to the new plant, and then settled down again at the end of the run. The resulting step response, Fig. 11, is slightly different than that of Fig. 4, but is essentially as good.

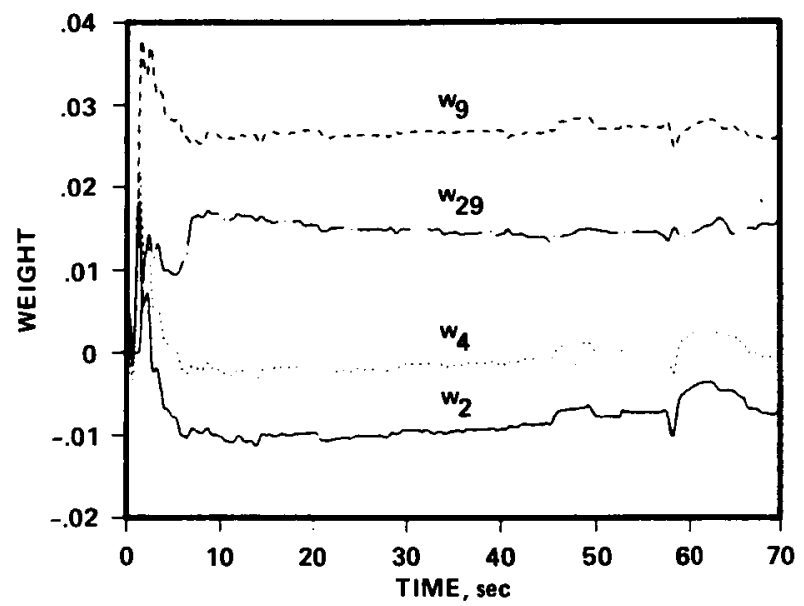

Fig. 10 Time history of filter weights, Example 3.

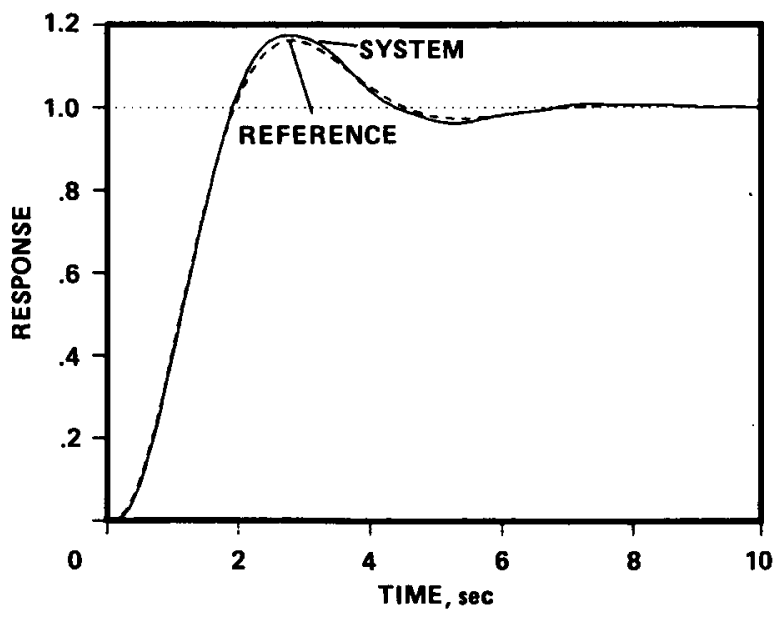

Fig. 11 Step response of adapted system and reference model $T(s)$, Example 3 .
Example 4: For this example, the numerator of $P(s)$ was increased by $50 \%$, from 25 to 37.5 . Thus, the gain of the plant was increased. Figure 12 shows the filter weights. The change in weights after $40 \mathrm{sec}$ is dramatic, but accurate, as shown by the resulting step response in $\mathrm{Fig} .13$.

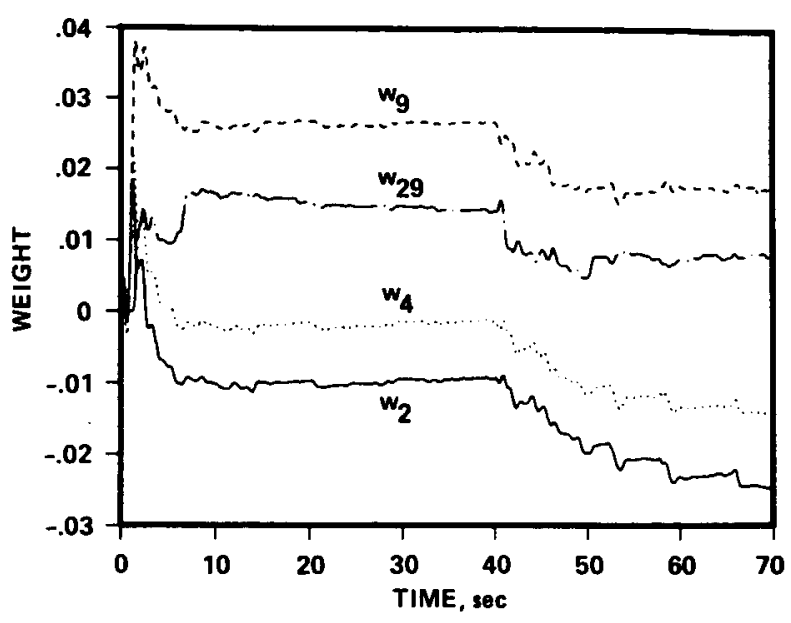

Fig. 12 Time history of filter weights, Example 4.

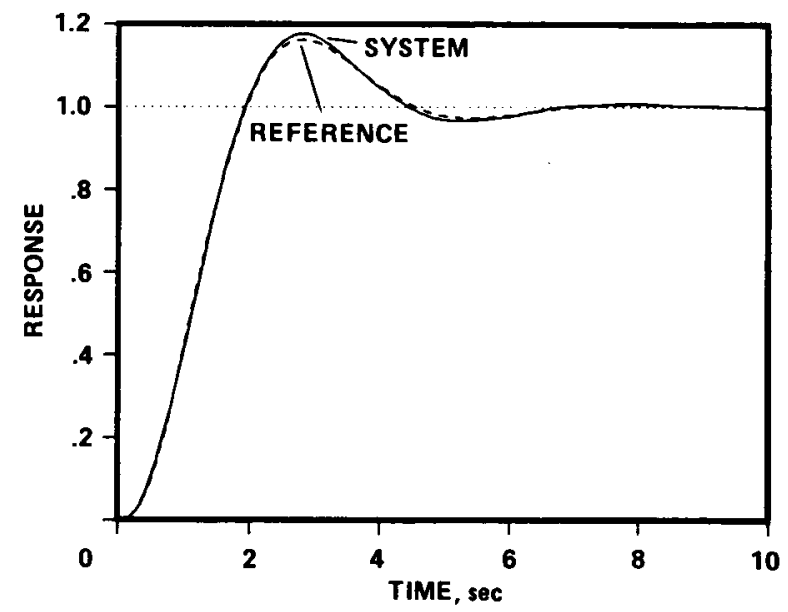

Fig. 13 Step response of adapted system and reference model $T(s)$, Example 4.

\section{The Effect of Noise}

It is difficult to design a "direct" adaptive controller which is not biased by noise. The reason is that the noise produces errors in the estimation process which then circulate through the system. When noise is included, the open-loop filter error is

$$
e=\frac{(M-G P) I}{1+M I} u-G n
$$

and the error gradient is 


$$
\frac{\partial e}{\partial w_{i}}=-g^{i}(z+n)=-g^{i} z_{m}
$$

Compare these with Eqs. (9) and (10). Since the filter error and the error gradient are multiplied together to form the change in filter weights, (Eq. (4)), terms in $n^{2}$ will appear, and therefore the filter weights will be biased. The filter error and the error gradient for the closed-loop state also include noise terms and similarly cause bias in the weights. Thus, bias in the filter weights is inevitable with this configuration. How seriously does the noiseinduced bias affect the performance of the system? The following examples provide indications. For both examples the noise signal is white noise and is uncorrelated with the input signal.

Example 5: Figure 14 was produced with a signal-to-noise ratio of 10 , and the system was

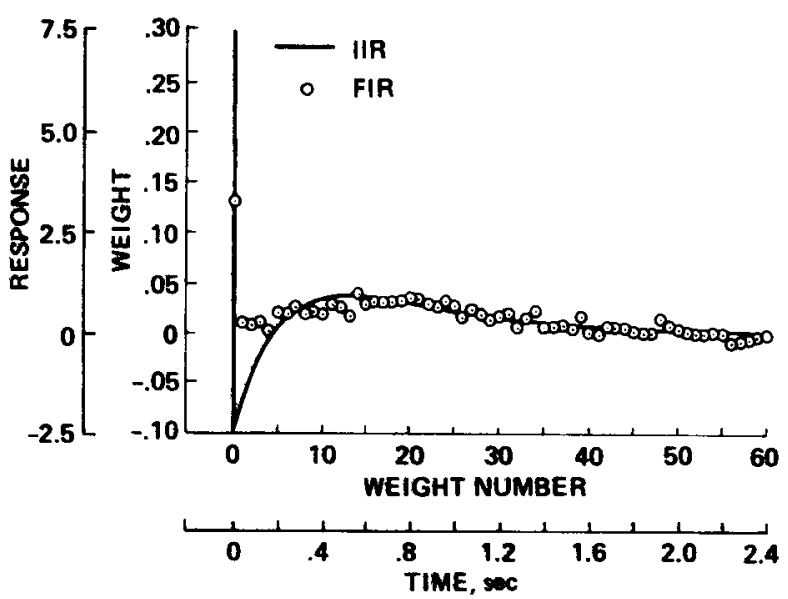

Fig. 14 Estimated FIR and IIR of $G(s)$, Example 5 .

started up in the closed-loop state with initial weights as in Eq. (24). Comparing this FIR to that of Fig. 3, we see some distortion of the filter weights because of noise. However, Fig. 15 shows that the step response of the adapted system is still good.

Example 6: Even in a very noisy system, with a signal-to-noise ratio of 2 , the step response is still quite close to that of the reference model as shown in Fig. 16. Although the system is biased in the presence of noise, the degradation in performance is minor.

\section{Applications}

The preceding sections have demonstrated excellent performance of the adaptive scheme of Fig. 2 with regard to initial adaptation to an unknown plant, tolerance to noise, and response to changes in plant parameters. In the following, we

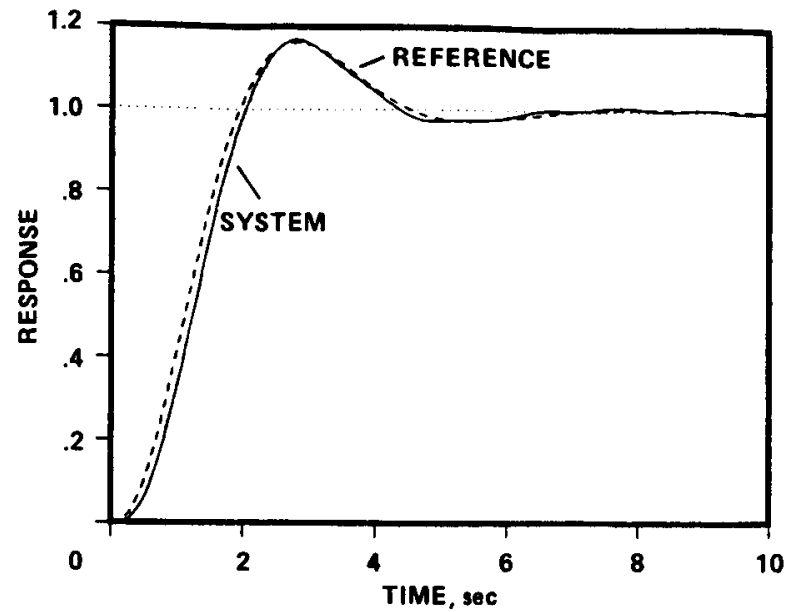

Fig. 15 Step response of adapted system and reference model $T(s)$, Example 5 .

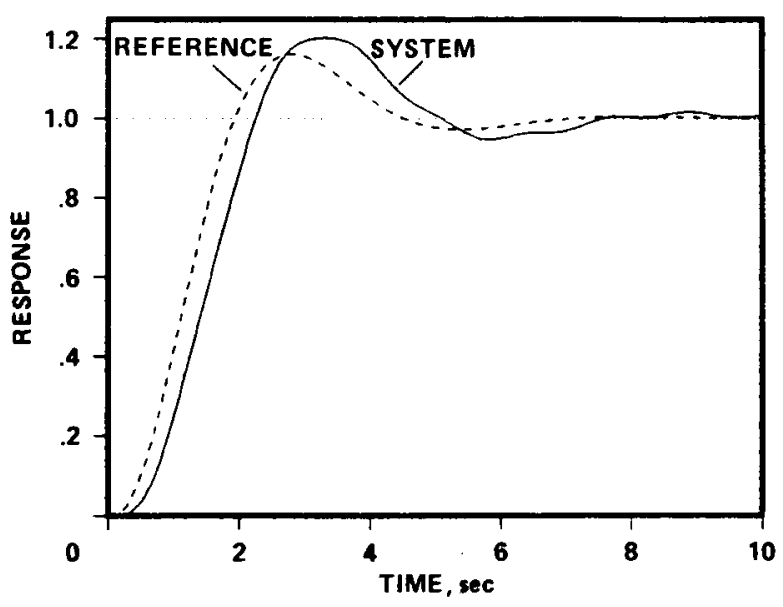

Fig. 16 Step response of adapted system and reference model $T(s)$, Example 6 .

discuss potential extensions and applications of this adaptive controller.

\section{In-Flight Adaptation}

For the control systems that go through large, but known, changes in operating environment and required performance (e.g., high-performance aircraft), a table of filter weights defining controllers for the different regimes could be created of $f-1$ ine and then used in flight as necessary. The advantages of using the adaptive controller of this paper for such systems are the same as have been shown in the examples, especially with regard to robustness and the ability to respond to variations in the plant.

\section{Unmodeled Modes}

In many cases, the plant is not completely unknown; a model is available, but is in some way incomplete. As long as a reasonable assumption 
can be made as to the order of the plant, so that the chosen reference model produces a realizable controller, one can take advantage of the partial knowledge by designing a controller that will make the known plant behave as desired and then let an adaptive controller handle the unknown portions of the plant.

One possible configuration with a controller with nominal and adaptive parts is shown in

Fig. 17, where the two parts are in parallel. To demonstrate the use of this configuration we use the following example.

Example 7: Let the actual plant be

$$
P(s)=\frac{10}{s^{2}+2 s+25}
$$

Let the known, or nominal, plant be

$$
P_{n}(s)=\frac{10}{s(s+2)}=\frac{10}{s^{2}+2 s}
$$

Equations (28) and (29) can be thought of as a mass/spring/damper system where the spring is unknown. The reference model, $M(s)$, is chosen as

$$
M(s)=\frac{175}{s^{2}+15 s+81}
$$

The resulting nominal controller is

$$
G_{n}(s)=\frac{M(s)}{P_{n}(s)}=\frac{17.5 s(s+2)}{s^{2}+15 s+81}
$$

The adaptive controller, G, should adjust so that

$$
\left[G(s)+G_{n}(s)\right]=\frac{M(s)}{P(s)}
$$

which results in

$$
G(s)=\frac{437.5}{s^{2}+15 s+81}
$$

Figure 18 shows the step response of the system with the nominal controller only, and that of the desired closed-loop reference model for comparison. The response is poor. The undesired oscillation originates from the unaccounted for spring forces. When the adaptive controller, G, is included, the response is much better as shown in Fig. 19. Thus, the adaptive controller is effective in compensating for the imperfections of the nominal controller.

\section{Fine Tuning}

As an extension of the concept illustrated in the previous example, the adaptive scheme can also be used to "fine tune" existing control systems. The plant, $P(s)$, of Fig. 2, can actually be an entire system of process and local controller. The local controller is designed to obtain a desired system performance, but because of inaccuracies in modeling and implementation, there is some degradation in system performance. The adaptive controller can then be added on as an additional loop of the system that will improve the performance to the desired level.

A potential application of this idea is in robotics. The equations of motion of a robot arm are generally well known, and straightforward control algorithms have been designed. 10 However, there is often uncertainty in the measurement of some of the physical characteristics $(\mathrm{e} . \mathrm{g}$., inertias and link lengths) that causes the performance to fall below expectations. The present adaptive scheme could be used for fine tuning of such a robot controller. It has also been suggested that the drive systems of robots need to be well modeled and these models be used in the development of robot controllers. 11 Some of the proposed models are very similar to the "unknown" plants

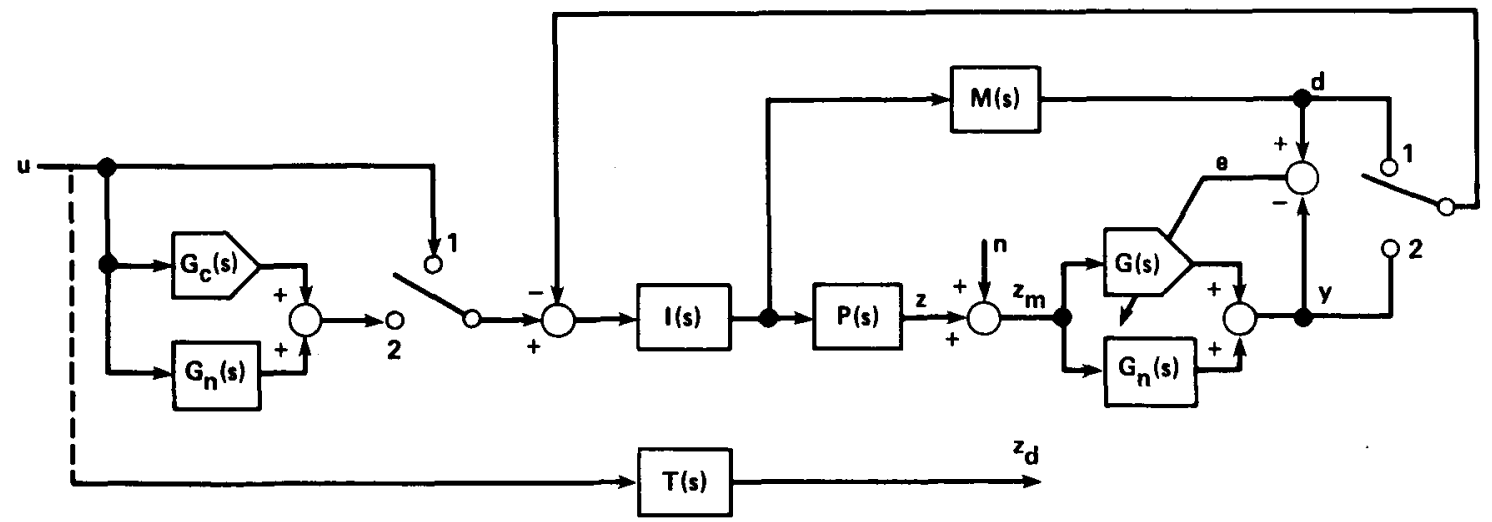

Fig. 17 A modified system configuration which includes a nominal as well as an adaptive controller. 


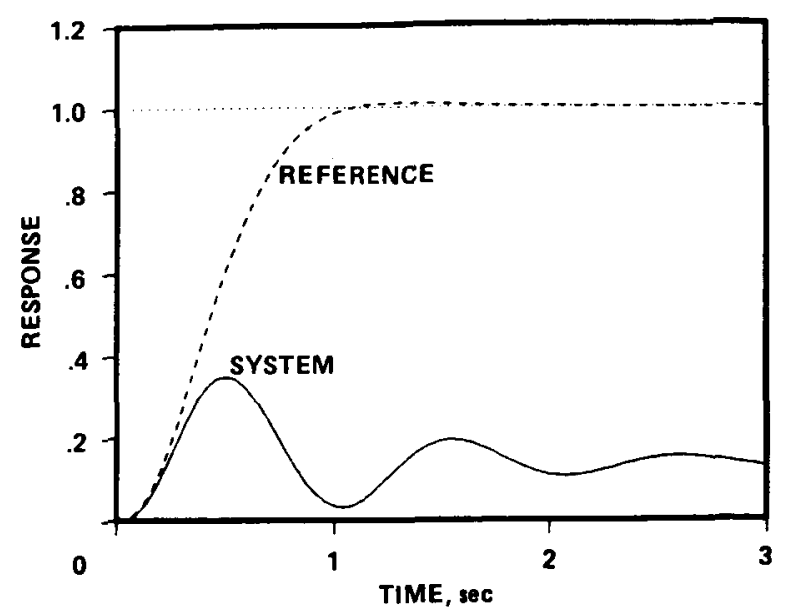

Fig. 18 Step response of system with nominal controller only and that of the reference model $\mathrm{T}(\mathrm{s})$, Example 7 .

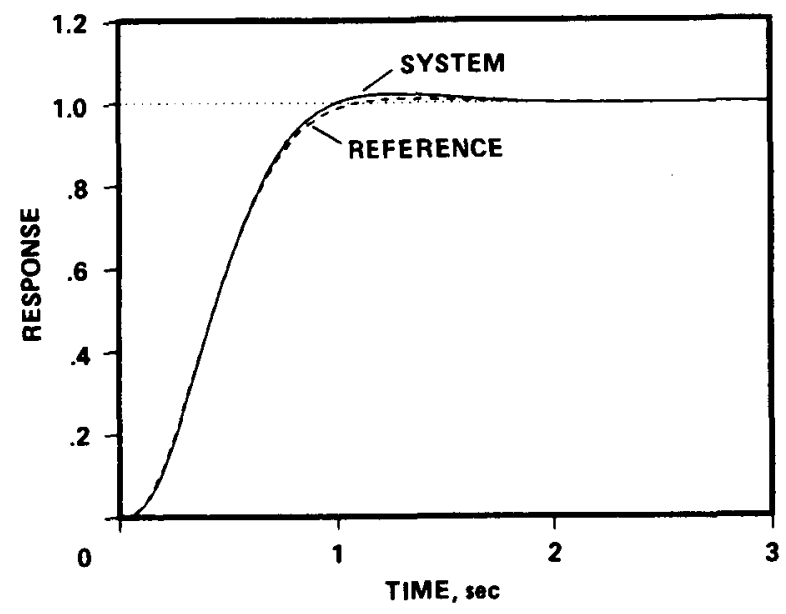

Fig. 19 Step response of adapted system and reference model $T(s)$, Example 7 .

used in our simulations, and the present adaptive scheme could be used to achleve drive system response that eases the development of such robot controllers.

\section{Concluding Remarks}

It has been shown that the impulse response modeling approach used in the development of the closed-loop model reference adaptive control scheme presented in this paper provides excellent overall performance. The scheme adapts well to an unknown plant and can converge in some cases without the need of a start-up scheme, continues to adapt to changes in plant behavior, and tolerates large amounts of nolse. Since the only essential a priorl knowledge of the plant that is needed is that it be stable and minimum phase, this concept could be extremely useful in control situations when it is difficult to determine an accurate model of the plant. The scheme may also find uses for the control of partially known plants, for the "fine tuning" of systems that already have acceptable control performance, and for the suppression of parasitic modes.

\section{References}

${ }^{1}$ Morse, A. S., "Global Stability of Parameter Adaptive Control Systems," IEEE Trans. on Automatic Control, Vol. AC-25, No. 3, June 1980, pp. 433-440.

${ }^{2}$ Astrom, K. J., "Theory and Applications of Adaptive Control--A Survey," Automatica, Vol. 19, No. 5 , Sept. 1983, pp. 471-486.

${ }^{3}$ Kreisselmeier, Gerhard and Anderson, Brian, D. 0., "Robust Model Reference Adaptive Control," IEEE Trans. on Automatic Control, Vol. AC-31, No. 2, Feb. 1986, pp. 127-133.

${ }^{4}$ Rohrs, C. E., Valvani, L., Athans, M., and Stein, G., "Robustness of Continuous-Time Adaptive Control Algorithms in the Presence of Unmodeled Dynamics," IEEE Trans. on Automatic Control, Vol. AC-30, No. 9, Sept. 1985, pp. 881-889.

${ }^{5}$ Richalet, J., Rault, A., Testud, J. L., and Papon, J., "Model Predictive Heuristic Control: applications to Industrial Process," Automatica, Vol. 14, Sept. 1978, pp. 413-428.

$6_{\text {Widrow, Bernard and Walach, Eugene, "Adap- }}$ tive Signal Processing for Adaptive Control," presented at IFAC Workshop on Adaptive Systems in Control and Signal Processing, June 1983.

7Widrow, B., McCool, J. M., Larimore, M. G., and Johnson, Jr., C. R., "Stationary and Nonstationary Learning Characteristics of the LMS Adaptive Filter," Proceedings of the IEEE, Vol. 64, No. 8, Aug. 1976, pp. 1151-1162.

${ }^{8}$ Merhav, S. J., "Design of Adaptive Control Systems by Means of Self Adjusting Transversal Filters," NASA TM 86843, 1986.

${ }^{9}$ Goodwin, Graham C. and Sin, Kwai Sang, Adaptive Filtering Prediction and Control, PrenticeHall, Inc., Englewood Cliffs, New Jersey, 1984, Chs. 7 and 9 .

${ }^{10}$ Craig, John J., Robotics: Mechanics and Control, Addison-Wesley, Reading, MA, 1986.

${ }^{11}$ Good, M. C., Sweet, L. M., and Strobel, K. L., "Dynamic Models for Control System Design of Integrated Robot and Drive Systems," Journal of Dynamic Systems, Measurement, and Control, Vol. 107, March 1985, pp. 53-59. 


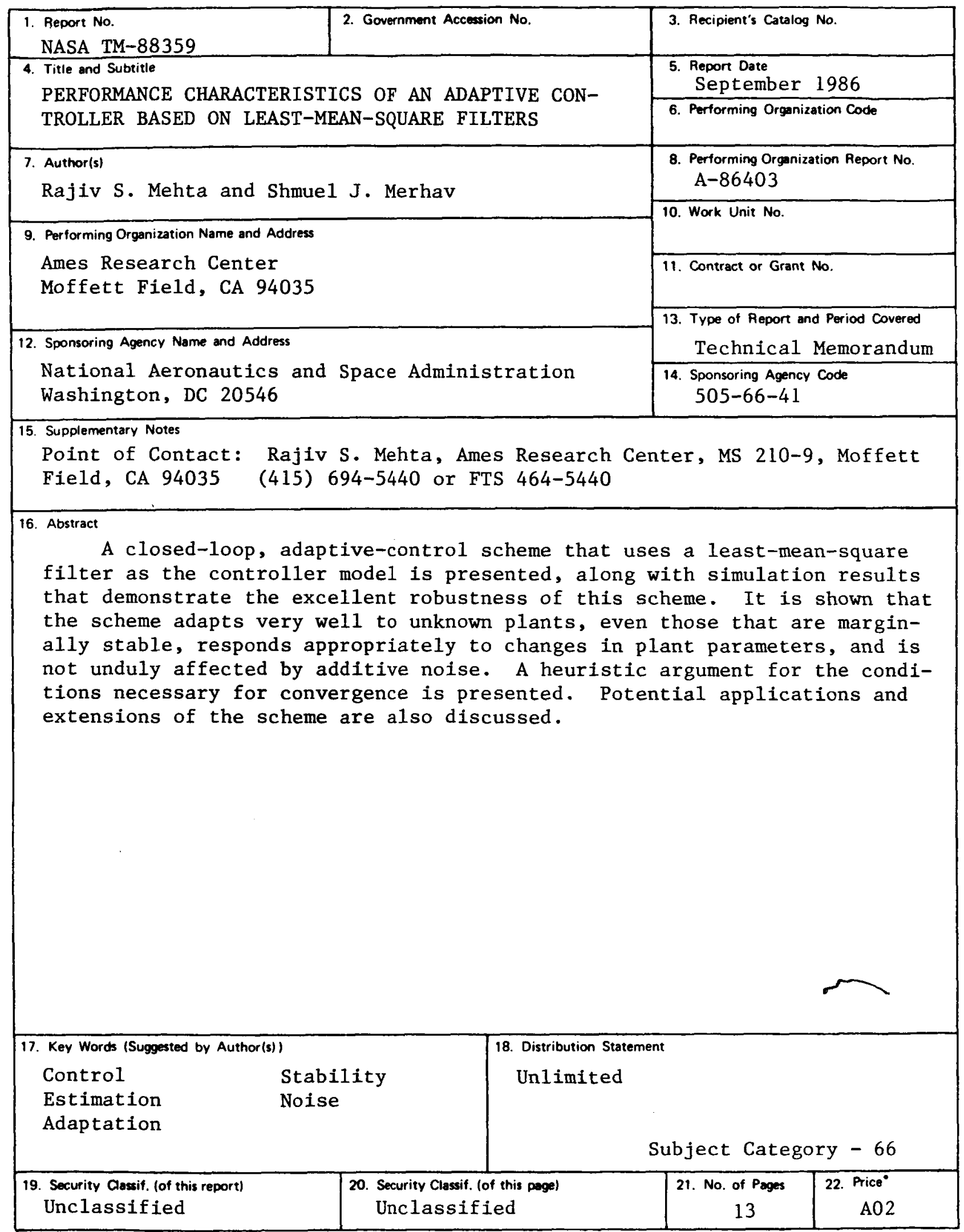

- For sale by the National Tochnical Information Service, Springfield, Virginia 22161 